\title{
Localization of Eosinophilic Esophagitis from H\&E stained images using multispectral imaging
}

\author{
Pinky A Bautista*, Yukako Yagi \\ From The 10th European Congress on Telepathology and 4th International Congress on Virtual Microscopy \\ Vilnius, Lithuania. 1-3 July 2010
}

\begin{abstract}
This study is an initial investigation on the capability of multispectral imaging to capture subtle spectral information that would enable the automatic delineation between the eosinophilic esophagitis and other eosin stained tissue components, especially the RBCs. In the method, a principal component analysis (PCA) was performed on the spectral transmittance samples of the different tissue components, excluding however the transmittance samples of the eosinophilic esophagitis. From the average spectral error configuration of the eosinophilic esophagitis transmittance samples, i.e. the difference between the actual transmittance and the estimated transmittance using $m$ PC vectors, we indentified two spectral bands by which we can localize the eosinophils. Initial results show the possibility of automatically localizing the eosinophilic esophagitis by utilizing spectral information.
\end{abstract}

\section{Background}

Eosinophils are type of white blood cells that are important part of the immune system. They are present in small amount in the intestine and blood but not normally in the esophagus. Infiltration of eosinophils into the esophagus could result to conditions such as eosinophilic esophagitis (EE) and gastroesophageal reflux. Since eosinophilic esophagitis and gastroesophageal reflux exhibit similar clinical and histology features studies have been conducted to determine the distinguishing features between the two conditions [1-3] where the number of eosinophil infiltration was found to be one of the indicative features of eosinophilic esophagitis. In [3] pathologists followed three methods to evaluate the infiltration of eosinophils: (1) subjective evaluation of the presence of eosinophils in the entire hematoxylin and eosin (H\&E) stained histology section where semiquantitative scoring was applied; (2) the eosinophils were counted in 5 high power fields (HPF, x400) and the average was calculated; and (3) eosinophil density was evaluated by counting the eosinophils in the mucosa of the entire histological section and measuring the area.

\footnotetext{
* Correspondence: pbautista@partners.org
Department of Pathology, Massachusetts General Hospital, Harvard Medical

* Correspondence: pbautista@partners.org
Department of Pathology, Massachusetts General Hospital, Harvard Medical School Boston, MA,USA
}

(c) 2011 Bautista and Yagi; licensee BioMed Central Ltd. This is an open access article distributed under the terms of the Creative

The consistency of the results in the subjective and manual approach of assessing the degree the eosinophils infiltration in the methods just mentioned can be improved if appropriate digital processing is applied to the images.

Using the spectral colour of an image pixel for automatic object classification or segmentation can be considered simple if only there is an obvious spectral colour difference between the objects of interest and the background objects. From an H\&E stained tissue slide the eosinophils appear red to pink similar to other connective tissue components such as the red blood cells (RBC). Thus there is a challenge in using the RGB colour vector as feature variable for the classification or segmentation of eosinophils.

Multispectral imaging is popularly applied to remote sensing applications, but it has gained significant attentions from researchers in various fields. The technology has been studied for accurate colour reproduction [4], colour enhancement [5,6], digital staining [7], and others. A multispectral imaging system employs more than $3(\mathrm{~N}>3)$ narrowband filters which result to greater spectral sensitivity compared to the conventional RGB imaging system which utilizes 3 broadband filters. The capability of multispectral imaging to delineate tissue 
structures that are closely similar in their spectral colour have been shown in [8]. In this paper we proposed a method to effectively visualize, detect and segment the eosinophils using information from the multispectral images of $H \& E$ stained tissue images, particularly using the spectral error between the original spectral transmittance of a pixel and its estimated transmittance which is calculated by using $m$ PC vectors.

\section{Materials and methods} Imaging system

The microscopic multispectral imaging system that we used in our experiment to capture the H\&E stained multispectral images is shown in fig. 1. Attached to the conventional microscope is a multispectral filter with spectral sensitivity in the visible spectrum, i.e. $400 \mathrm{~nm}$ $700 \mathrm{~nm}$. Using our in-house software we can capture multispectral images at $5 \mathrm{~nm}$ bandwidth across the visible spectrum and saved the $1034 \times 1050$ pixel images in TIFF format with 16-bit colour depth.

\section{Multispectral images}

We captured 10 sets of $H \& E$ stained images with the microscope objective lens set at 20x. In [6,8] 16 multispectral bands were used to enhance regions of collagen fiber to differentiate them from muscle fiber wherein the two tissue structures share similar colorimetric attributes in an H\&E stained image. In our experiment the original 55-band multispectral (MS)

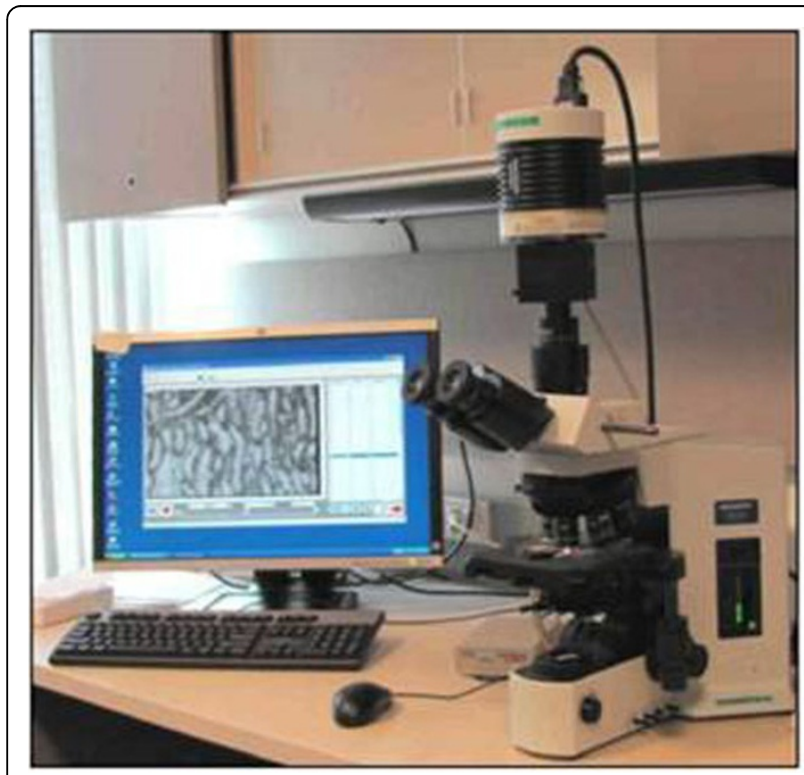

Figure 1 The microscopic multispectral imaging system that was used to capture the multispectral images of H\&E stained esophagus tissue in the experiment. Attached to the conventional microscope is a multispectral filter with sensitivity from $400 \mathrm{~nm}$ to $720 \mathrm{~nm}$. images were reduced to 14-band MS images by taking the spectral average of the MS pixel at four contiguous bands. Table 1 illustrates the spectral sensitivity of the resulting bands.

\section{Spectral transmittance}

The $n$-band spectral transmittance of a pixel is calculated by taking the ratio between the signal value, $\mathbf{i}_{\mathrm{s}}$, of the specimen and that of glass, $\mathbf{i}_{\mathrm{g}}$ :

$$
\mathbf{f}=\frac{\mathbf{i}_{s}}{\mathbf{i}_{g}}
$$

where the entries of the Nx1 column vector $\mathbf{f}$ correspond to the spectral values at different wavelengths. We manually extracted the spectral samples for nucleus, cytoplasm, red blood cells (RBC), fiber, white area (the area in the image which is void of tissue) from the 5 images of the 10 sets of images that we captured. .

\section{Principal component analysis (PCA)}

The goal of PCA is to reduce the number feature variables while preserving the variance of the feature data. If we let $\mathbf{F}$ be an $n x q$ data matrix representing $q$ samples of an $n$-dimensional feature vector:

$$
F=\left[\begin{array}{ccccc}
f_{11} & f_{12} & \cdots & f_{1 q-1} & f_{1 q} \\
f_{21} & f_{22} & \cdots & f_{2 q-1} & f_{2 q} \\
\vdots & \vdots & \ddots & \vdots & \vdots \\
f_{n-11} & f_{n-12} & \cdots & f_{n-1 q-1} & f_{n-1 q} \\
f_{n 1} & f_{n 2} & \cdots & f_{n q-1} & f_{n q}
\end{array}\right],
$$

Table 1 The multispectral (MS) band numbers and their wavelength counterparts. The value of the spectral transmittance at MS band \#1 corresponds to the average transmittance value between $\lambda_{1}$ and $\lambda_{2} \mathrm{~nm}$

\begin{tabular}{cc}
\hline Multispectral band \# & Wavelength [nm] \\
\hline 1 & $450-465$ \\
2 & $470-485$ \\
3 & $490-505$ \\
4 & $510-525$ \\
5 & $530-545$ \\
6 & $550-565$ \\
7 & $570-585$ \\
8 & $590-605$ \\
9 & $610-625$ \\
10 & $630-645$ \\
11 & $650-665$ \\
12 & $670-685$ \\
13 & $690-705$ \\
14 & $710-720$ \\
\hline
\end{tabular}


then $n \times n$ covariance of the of the matrix $\mathbf{F}$ can be derived as follows [9]:

$$
\mathbf{C}=\frac{1}{q-1} \sum_{\mathrm{i}=1}^{q}\left(\mathbf{F}_{\mathrm{i}}-\overline{\mathbf{F}}\right)\left(\mathbf{F}_{\mathrm{i}}-\overline{\mathbf{F}}\right)^{\mathrm{T}}
$$

where $\overline{\mathbf{F}}$ is the mean vector of matrix $\mathbf{F}$ given by:

$$
\overline{\mathbf{F}}^{\mathrm{T}}=\frac{1}{q} \sum_{\mathrm{i}=1}^{q} \mathbf{F}_{\mathrm{i}}
$$

The diagonal elements of the covariance matrix, $c_{i i}$, denotes the variance of the data around the mean while the off diagonal elements, $c_{i j}$, express the degree of correlation between the $i t h$ and $j t h$ feature variables. From the covariance matrix $\mathbf{C}$ we can derive the eigenvectors and eigenvalues and by arranging the eigenvectors in descending order of their eigenvalues we can form an orthogonal basis having the first eigenvector containing the largest variance of the original data. The \% variance of the original data using the first $m$ eigen vectors can be explained by the ratio between the sum of $m$ and $n$ eigenvalues:

$$
\% \operatorname{var}=\frac{\sum_{i=1}^{m} \lambda_{i}}{\sum_{i=1}^{n} \lambda_{i}} x 100,
$$

where $\lambda_{i}$ is the ith eigenvalue. If most of the variance in the original data is contained in the first $m$ eigenvectors, say $99.99 \%$, a reconstruction of the original data can be done by forming a linear combination of these vectors:

$$
\hat{\mathbf{f}}=\overline{\mathbf{F}}+\sum_{\mathrm{i}=1}^{m} \alpha_{\mathrm{i}} \mathbf{v}_{\mathrm{i}}
$$

where $\alpha_{\mathrm{i}}$ is the PC coefficient and $\mathbf{v}_{\mathrm{i}}$ is the ith eigenvector.

\section{Spectral error}

The reconstruction error that results from the application of eqn. 6 largely depends on the accurate estimation of the data covariance matrix $\mathbf{C}$ which in turn is governed by the sufficiency of the data samples in $\mathbf{F}$. If the feature variance of a sample is captured in the data matrix $\mathbf{F}$ fewer eigenvectors are needed to obtain smaller error in the reconstruction of such sample feature.

PCA is also a technique used to address the estimation of $n$ dimensional spectral data by using $m<n$ eigenvectors. If we consider $\mathbf{F}$ as the data matrix representing the spectral samples of some objects such that $\mathbf{f}_{\mathrm{k}}$ represents the spectrum of a particular object and $\mathbf{f}_{\mathrm{k}}$ its estimate derived from eqn. 6 then we can write the spectral error as follows:

$$
\mathbf{e}_{\mathrm{k}}=\mathbf{f}_{\mathrm{k}}-\hat{\mathbf{f}}_{\mathrm{k}}
$$

where $\mathbf{e}_{\mathrm{k}}$ is an $n$ dimensional column vector. The magnitude of the error in eqn.7 is a function of the estimation of $\hat{\mathbf{f}}_{\mathrm{k}}$. Consider that there are $c$ classes of objects that are identified from an image but only $c-1$ of these classes are represented with spectral samples in the data matrix $\mathbf{F}$, then for a given $m$ eigenvectors the spectral errors are smaller for objects that belong to the first $c-1$ classes compared to objects that belong to the $c$ th class.

\section{Detection and segmentation using the spectral error}

The configuration of $\mathbf{e}_{\mathrm{k}}$, i.e. the wavelengths at which the error peaks, is a function of the spectral attributes of the object. If we could identify two wavelengths $r$ and $s$ at which the spectral error of the $c$ th class has the highest positive and negative peaks while the $c-1$ classes of objects experience an almost zero spectral error at these wavelengths it is possible to segment the objects in $c$ th class by taking the difference between the spectral errors at these wavelengths:

$$
\mathrm{d}=\mathrm{e}_{\mathrm{r}}-\mathrm{e}_{\mathrm{s}}
$$

where $e_{r}$ is the highest positive peak and $e_{s}$ the negative peak with the largest magnitude. To segment objects belonging to the cth class a threshold can be applied to the result of eqn. 8

$$
d_{c}= \begin{cases}0 & d<=0 \\ 1 & d>0\end{cases}
$$

Although in an H\&E stained slide the tissue structures are generally categorized as either acidophilic or basophilic each tissue structure has its own distinct spectral attributes due to its unique reactions to the chemical dyes. Hence the spectral error of a tissue component not represented in the data matrix $\mathbf{F}$ would likely exhibit peaks at certain wavelengths for a given $m$ eigenvectors; these wavelengths might be correlated to the absorption peaks of the dyes themselves. With these specific wavelengths identified it is possible to detect and segment such particular tissue component by applying appropriate thresholds. Furthermore translating the spectral error values at these wavelengths would also result to better visualization of the tissue component. 
The general processes involved in the localization of eosinophils are illustrated in the block diagram in fig. 2 . First the spectral transmittance of a pixel is calculated then its estimate is calculated using $m$ PC vectors that were previously identified in an off line experiment. The difference of the spectral errors between two bands is then calculated. Application of an appropriate threshold, eqn.9, segments the eosinophils.

\section{Results}

The objective of this paper is to detect and segment the eosinophils from H\&E stained esophagus tissue images. For the purpose of the experiment we collected 10 multispectral images from two different H\&E stained slides.

\section{Spectral residual-error}

The training set $\mathbf{F}$ consisted of over 3000 transmittance samples belonging to five tissue components: nucleus, cytoplasm, red blood cells (rbc), fiber and white area (area which does not contain any tissue). From this data set we calculated the covariance matrix $\mathbf{C}$ and derived the eigen vectors or principal component $(\mathrm{PC})$ vectors, and the eigenvalues. The plot in fig. 3 shows the $\%$ accumulated variance calculated from eqn. 5. It can be interpreted from the plot that with $5 \mathrm{PC}$ vectors the spectral errors of the five tissue components would reduce to zero.

The graph in fig. 4 illustrates the configurations of the 14-band average spectral errors of the 5 tissue components whose spectral samples were used to derive the covariance matrix that of eosinophils. We see from the graph that the exclusion of the eosinophil spectral samples from the training data results to larger spectral error magnitude and distinct peaks at certain wavelengths. For instance at band $7(570 \mathrm{~nm}-585 \mathrm{~nm})$ and band $10(630 \mathrm{~nm}-645 \mathrm{~nm})$ largest negative and positive peaks are observed.

\section{Detection and Segmentation of the eosinophils}

The wavelengths (or bands) at which the spectral errors of the eosinophils are the largest in magnitude were

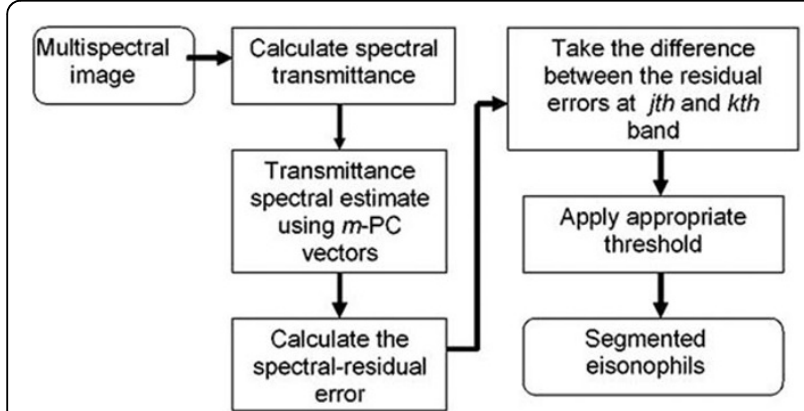

Figure 2 Block diagram showing the processes involve in the segmentation of eosinophils.

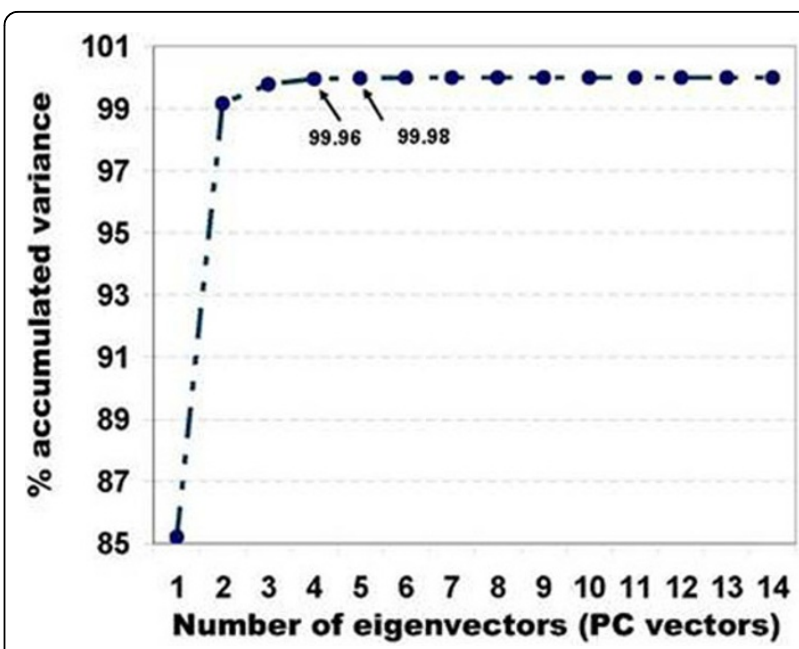

Figure 3 The \% accumulated variance for different number of eigenvectors (PC vectors). This plot has been generated using the spectral samples of nuclei, cytoplasm, red blood cells, fiber and white areas (areas which do not contain tissue). We can observe that with more number of $P C$ vectors the variance of the data samples is captured better.

identified from the spectral-error plot itself, fig. 5. After we have identified these wavelengths we converted the error values at these wavelengths to grey-level image to obtain better visualization of the eosinophils. Samples of the H\&E stained images, which were extracted from an esophagus tissue slide containing eosinophils, in their RGB format along with spectral error images at band 7 $(570 \mathrm{~nm}-585 \mathrm{~nm})$ and band $10(630 \mathrm{~nm}-645 \mathrm{~nm})$ are shown in fig. 6 . The eosinophils are stained pink to red, which is similar with other connective tissues such as the red blood cell. However from the error images such

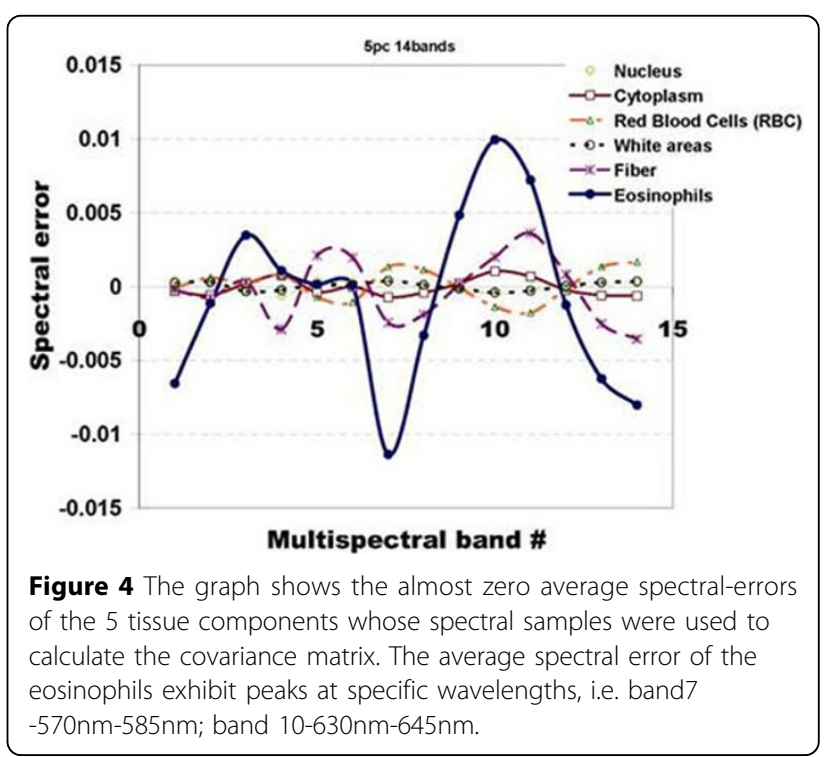




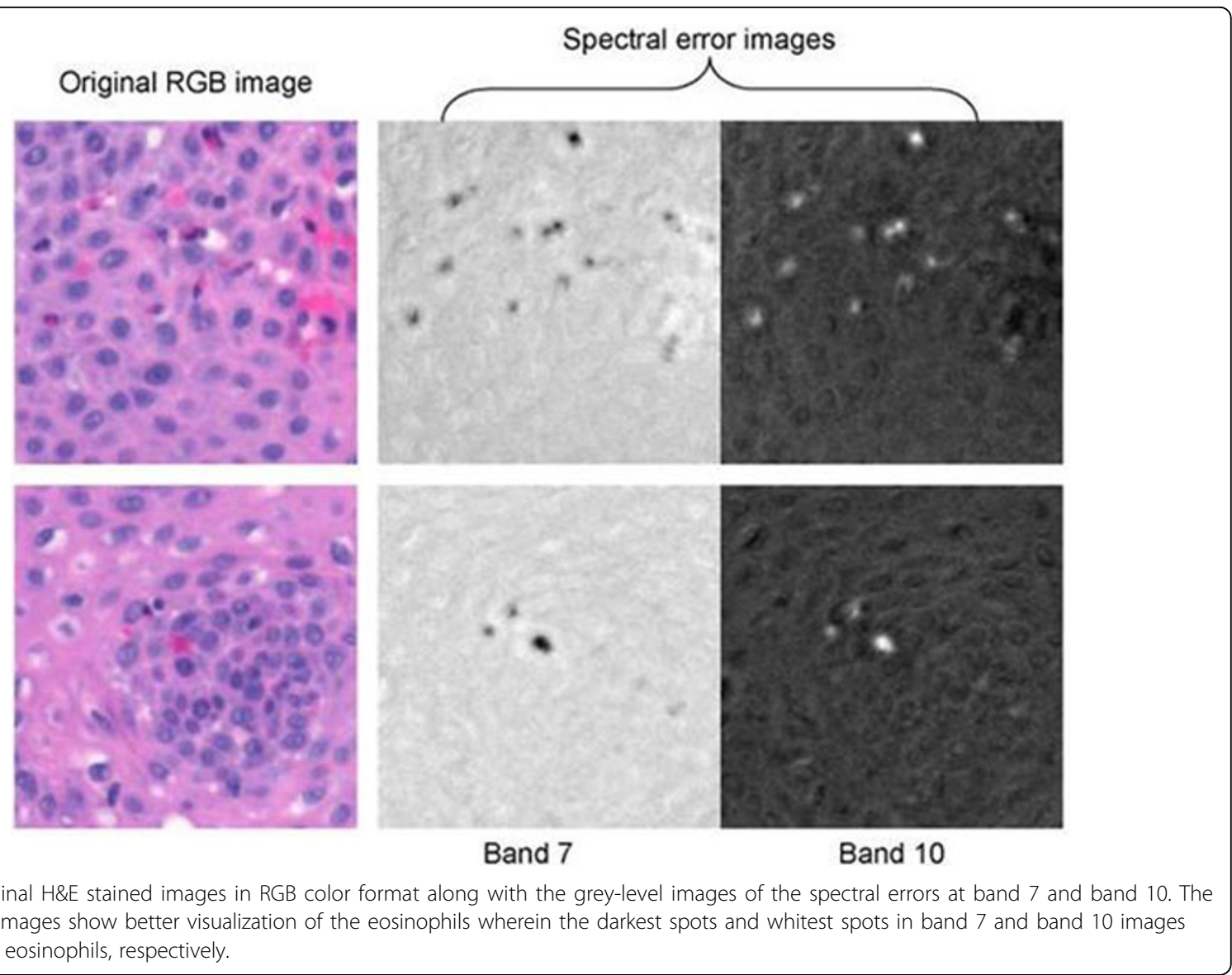

similarity is not visible - the eosinophils are distinctly marked in the images. In band 7 the eosinophils appear as black spots because they acquired the lowest spectral error at this band, refer to fig. 5. Since the spectral error of the eosinophils at band 10 is the highest they are the white spots in the error image at this band.

To segment the eosinophils the spectral errors at band 7 and band 10 were utilized. By implementing eqn. 8 and 9 where $e_{\mathrm{r}}$ correspond to the spectral error at band 10 and $\mathrm{e}_{\mathrm{s}}$ to the error at band 7, the eosinophils were successfully segmented. Figure 6 shows the result of the segmentation after applying morphological filter, i.e. dilation and erosion (edge detection using the canny algorithm as implemented in Matlab was applied to the results before they are overlaid on the original RGB color images). Here we see that the method was able to delineate between eosinophils and other similarly stained tissue components, i.e.RBC. There are however few eosinophils that were not detected by the process and this can be because (i) the spectral training samples was insufficient; (ii) the bandwidth of each spectral band is not narrow enough to capture minute changes in the spectral colours; and/or (iii) the number of spectral bands is not enough to capture the subtle spectral difference between eosinophils and other eosin stained tissue structures.

\section{Segmentation using RGB color information}

To determine the effectiveness of using RGB color information to segment the eosinophils we implemented a $k$-means clustering to the image containing both eosinophils and RBC. We set $k=6$ to represent all the tissue components that we have identified from the training images and this includes the eosinophils. The eosinophil areas that result from the clustering are shown in fig. 7 . Here we see that clustering the image pixels using the RGB color information failed to detect the difference between the eosinophils and the red blood cells; the mis-classified areas are indicated by the arrows.

\section{Discussion}

In a stained tissue slides, tissue structures which differ in their functionality can be stained with the same dye. The eosinophils and red blood cells (RBC), for instance, are both stained with eosin dye which made them share similar color attributes, i.e. pink to red. We have introduced a simple way to detect and segment the eosinophils using multispectral information particularly from the spectral errors of the 

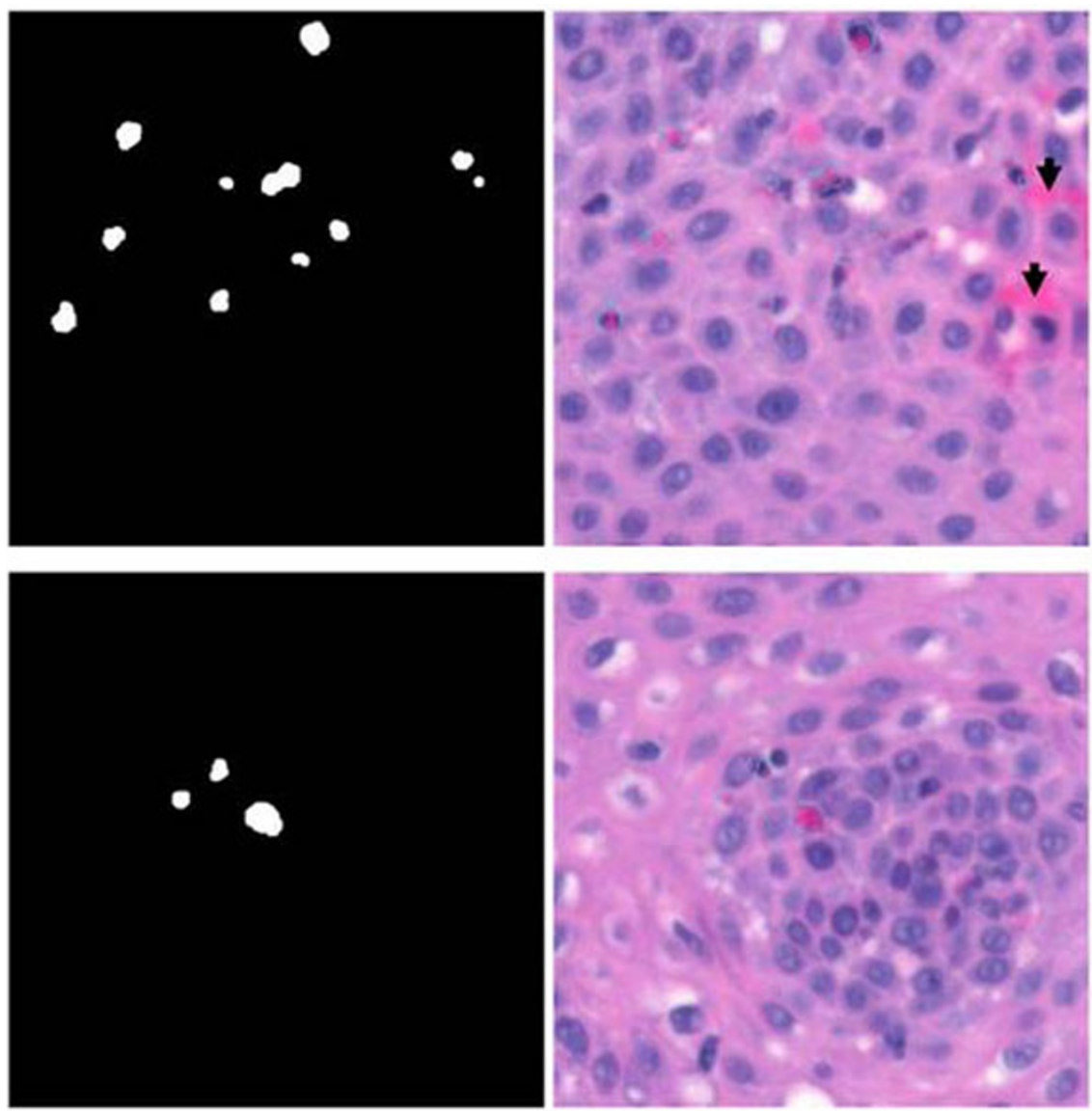

Figure 6 Segmentation results using multispectral information. The segmentation was done by taking the difference between the spectral errors at band 7 and band 10 and then applying the threshold specified by eqn.10; the images on the $2^{\text {nd }}$ column show the segmentation results overlaid on the original RGB images. It is worth noting that tissue structures (indicated by arrows) which acquired similar colorimetric attributes to eosinophils were not identified as eosinophils.

multispectral pixels. The utilization of spectral errors was first introduced in [5] to enhance abnormal skin areas. With modifications of the method the used of spectral error was applied to H\&E stained images to improve the colorimetric difference between collagen and muscle fiber [6,7]. Spectral error subtraction as a method to segment tissue components, which is currently being utilized to segment/detect eosinophils, was not addressed in both papers, however.

The effectiveness of utilizing multispectral information as opposed to RGB color information was shown in the segmentation results where the results using only the RGB color information was found inferior to the results from using multispectral information. An average plot of the 14-band spectral transmittance and RGB color values of $20 \mathrm{RBC}$ and eosinophil samples are shown in fig. 8. While we can see variations between the spectra of RBC and eosinophil in the whole visible spectrum, this is not directly observed from the RGB color plots where the difference is concentrated in the long wavelength range, i.e. red channel. That is the two structures

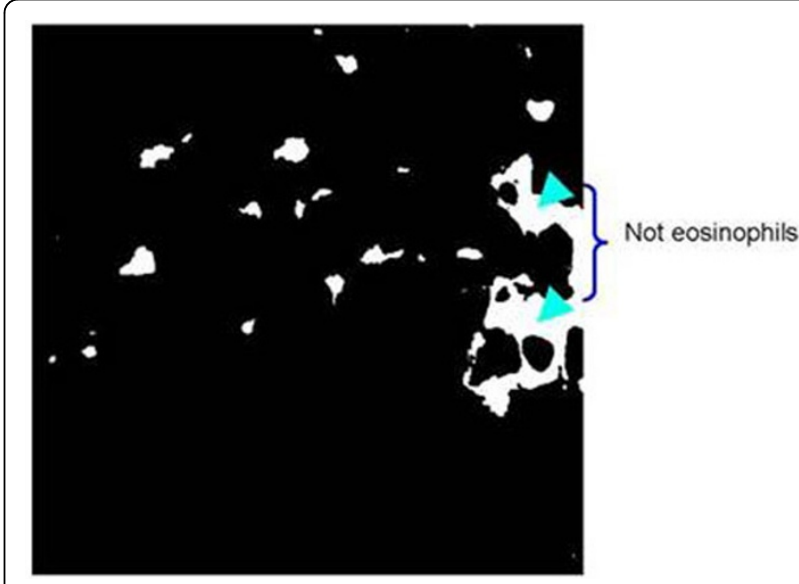

Figure 7 Segmentation result by $k$-means $(k=6)$ clustering using the RGB color information showing the cluster group containing the eosinophils.If we refer this result to the original RGB color image (fig. 7, topmost, $2^{\text {nd }}$ column) we can we see that tissue structures which are not eosinophils at all were also identified as eosinophils in the result, particularly the areas indicated by arrows. 
likely differ in their red color intensity in which case minute shift in the color cannot be detected.

The peaks exhibited by the spectral error of eosinophil in fig. 4 can be correlated to the dye absorption peaks. Plots of the hematoxylin and eosin 14-band dye absorptions are shown in fig. 9; the spectral error of the eosinophil is scaled and superimposed on the plot. We can observe that the peaks at band 7 and band 10 correlate to the hematoxylin absorption peak. The correspondence of the spectral error peak to the absorption peak of hematoxylin dye could imply that the difference between RBC and eosinophils can be accounted to their absorption of hematoxylin dye. More experiments however, are still needed to further justify this claim.
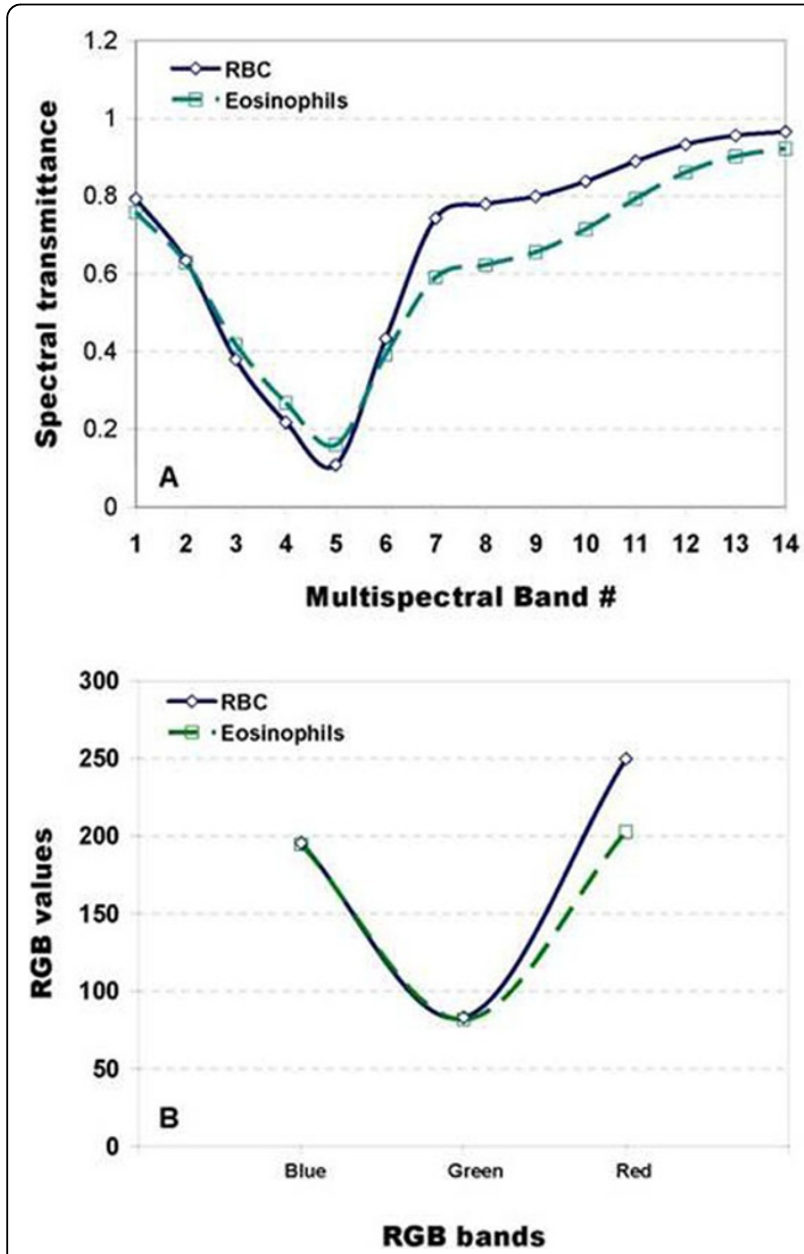

Figure 8 Panel A and Panel B show graphs of the characteristic 14band spectral transmittance and 3-band (RGB) spectral colour of the eosinophils and RBC (red blood cells), respectively. In panel A we can see differences between the RBC and eosinophil in their spectral configurations but their RGB colour values do not significantly differ as illustrated in Panel B, except in their red colour intensity which can vary depending on the staining.

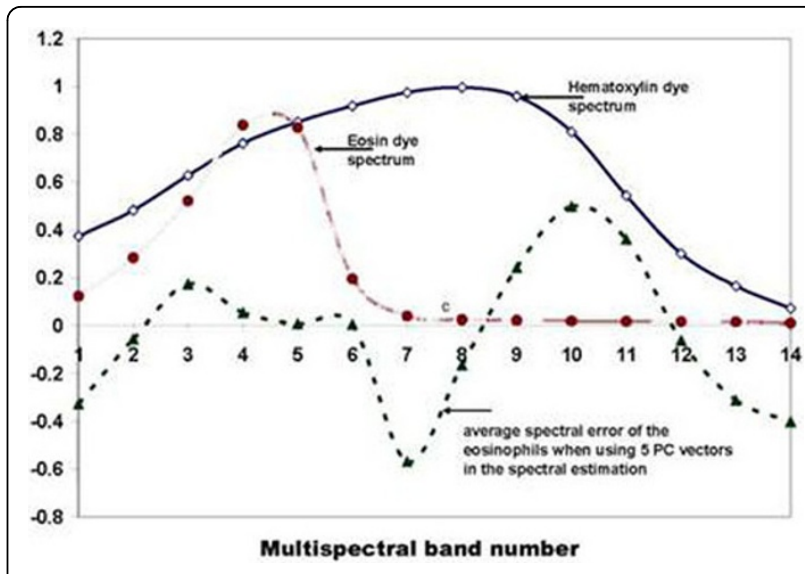

Figure 9 The eosin and hematoxylin dye absorption spectra and the average spectral error of the eosinophils; the spectral error of the eosinophils is scaled to have a clearer illustration. We can observe that the error peaks of the eosinophils correspond to the absorption peak of the hematoxylin dye. This could imply that the difference between eosinophils and other tissue structures which are stained similarly lies on their absorption of the hematoxylin dye.

\section{Conclusions}

In this paper we have addressed the detection and segmentation of eosinophils from an H\&E stained slide of an esophagus tissue using multispectral information, particularly using the spectral error difference between two specific bands that were identified from the plot of the spectral error itself. The results of the experiment show that with multispectral information it could be possible to classify tissue structures with very similar staining attributes which is difficult with the conventional RGB color information.

Quantification of eosinophils in H\&E stained esophagus tissue images is helpful in identifying eosinophilic esophagitis from gastroesophageal reflux. The segmentation of eosinophils presented in this paper can be utilized as a first step in the quantification process. Moreover, the proposed method could also be useful to segment other tissue structures which are stained similarly by simply excluding the spectral samples of such structures in the derivation of the eigenvectors (PC vectors) that are used to estimate the spectral transmittance of a multispectral pixel.

\section{Acknowledgment}

The authors thank Dr. Hongki Yoo, Ph.D and Dr. Guillermo J. Tearney, M.D, Ph.D of the Wellman Center for Photomedicine at Massachusetts General Hospital, Boston ,USA for the H\&E stained slides.

This article has been published as part of Diagnostic Pathology Volume 6 Supplement 1, 2011: Proceedings of the 10th European Congress on

Telepathology and 4th International Congress on Virtual Microscopy. The full contents of the supplement are available online at

http://www.diagnosticpathology.org/supplements/6/S1.

Authors' contributions

The authors contributed equally. 


\section{Competing interests}

MGH received financial grant from Olympus Co., Japan, but not for this project.

Published: 30 March 2011

\section{References}

1. Jeremy RParfitt, James CGregor, et al: Eosinophilic Esophagitis in Adults: disguishing features from gastroesophgeal reflux disease: A study of 41 patients. Modern Pathology 2006, 19:90-96.

2. Genevay M, Rubbia-Brandt L, Rougemont AL: Do Eosinophil Numbers Differentiate Eosinophilic Esophagitis from Gastroesophageal Reflux Disease? Arch Pathol Lab Med 2010, 134:816-825.

3. Piazuello MB, Camargo MC, Mera MR, et al: Eosinophils and mast cells in chronic gastritis: possible implications in carcinogensis. Human Pathology 2008, 39:1360-1369.

4. Yamaguchi $\mathrm{M}$, et al: Color image reproduction based on the multispectral and multiprimary imaging: Experimental evaluation. Proc. SPIE 2002, 4663:15-26.

5. Mitsui $M$, et al: Color enhancement in multispectral images using the Karhunen-Loeve transform. Optical Review 2005, 12:60-75.

6. Bautista PA, et al: Multispectral image enhancement for H\&E stained pathological tissue specimen. Proc. SPIE 2008, 6918.

7. Bautista PA, et al: Digital staining of Unstained Patnological Tissue Samples through Transmittance Classification. Optical Review 2005, 12:7-14.

8. Bautista PA, et al: A Multispectral Image Enhancement Approach to Visualize Tissue Structures. Arch Pathol Lab Med 2008, 1149.

9. Tzeng DY, Berns R: A Review of Principal Component Analysis and Its Applications to Color Technology. Color Research and Application 2005, 30:85-98.

doi:10.1186/1746-1596-6-S1-S2

Cite this article as: Bautista and Yagi: Localization of Eosinophilic Esophagitis from H\&E stained images using multispectral imaging. Diagnostic Pathology 2011 6(Suppl 1):S2.

\section{Submit your next manuscript to BioMed Central and take full advantage of:}

- Convenient online submission

- Thorough peer review

- No space constraints or color figure charges

- Immediate publication on acceptance

- Inclusion in PubMed, CAS, Scopus and Google Scholar

- Research which is freely available for redistribution

Submit your manuscript at www.biomedcentral.com/submit
Biomed Central 